\title{
Spatial “Artistic" Networks: From Deconstructing Integer-Functions to Visual Arts
}

\author{
Ernesto Estrada ${ }^{1}{ }^{1}$ and Puri Pereira-Ramos ${ }^{2}$ \\ ${ }^{1}$ Department of Mathematics \& Statistics, University of Strathclyde, 26 Richmond Street, Glasgow G1 1HQ, UK \\ ${ }^{2}$ PeRArt Studio, Murgas 4, 15822 A Coruna, Spain \\ Correspondence should be addressed to Ernesto Estrada; ernesto.estrada@strath.ac.uk
}

Received 19 September 2017; Accepted 9 December 2017; Published 17 January 2018

Academic Editor: Gerard Olivar-Tost

Copyright (C) 2018 Ernesto Estrada and Puri Pereira-Ramos. This is an open access article distributed under the Creative Commons Attribution License, which permits unrestricted use, distribution, and reproduction in any medium, provided the original work is properly cited.

\begin{abstract}
Deconstructivism is an aesthetically appealing architectonic style. Here, we identify some general characteristics of this style, such as decomposition of the whole into parts, superposition of layers, and conservation of the memory of the whole. Using these attributes, we propose a method to deconstruct functions based on integers. Using this integer-function deconstruction we generate spatial networks which display a few artistic attributes such as (i) biomorphic shapes, (ii) symmetry, and (iii) beauty. In building these networks, the deconstructed integer-functions are used as the coordinates of the nodes in a unit square, which are then joined according to a given connection radius like in random geometric graphs (RGGs). Some graph-theoretic invariants of these networks are calculated and compared with the classical RGGs. We then show how these networks inspire an artist to create artistic compositions using mixed techniques on canvas and on paper. Finally, we call for avoiding that the applicability of (network) sciences should not go in detriment of curiosity-driven, and aesthetic-driven, researches. We claim that the aesthetic of network research, and not only its applicability, would be an attractor for new minds to this field.
\end{abstract}

\section{Introduction}

There are multiple connections between networks and the visual arts. The study of graph drawing is an old topic in computer sciences and one of its main goals is the representation of networks in aesthetically appealing ways [1,2]. In modern network theory, there have been extraordinary advances in the visualization of giant complex networks, which can be considered as pieces of art by themselves [3]. A different direction is the use of networks as an artistic mean of expression. The artistic work of Tomás Saraceno is an example of this kind of symbiosis where the author has used spider webs to create a universe of expressions [4]. Other artists melt networks into evocative images of the real-world to produce artistic designs. This is the case of the artist J. K. Rofling who has produced many of these symbiotic images [5]. Some examples of the work of J. K. Rofling are illustrated in Figure 1.

Here, we explore a different approach to connect networks and the visual arts. Essentially, we start from the construction of spatial networks based on simple rules, namely, the location of points in a unit square. However, the coordinates of these points are generated by a mathematical transformation of integer numbers that generates artistic patterns on the plane. The inspiration for such transformation of integers and functions based on them comes from the "poststructuralist" school of philosophy and literary criticism known as deconstruction. This school started in the late 1960 after the influential book De La Grammatologie (1967) by the French philosopher Derrida [6]. This school of philosophical thinking influenced any areas of intellectual and creative activity including novels, poetry, architecture, the fine arts, and music. In architecture in particular, the term "deconstructivism" was adopted since the end of the 1980s [7]. According to Derrida this architectural style "is not simply the technique of an architect who knows how to deconstruct what has been constructed but a probing which touches upon the technique itself, upon the authority of the architectural metaphor and thereby constitutes its own architectural rhetoric" (cited by Hoteit in [7]). 


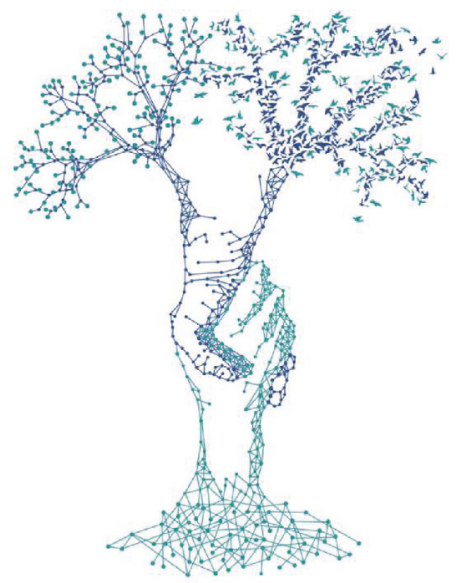

(a)

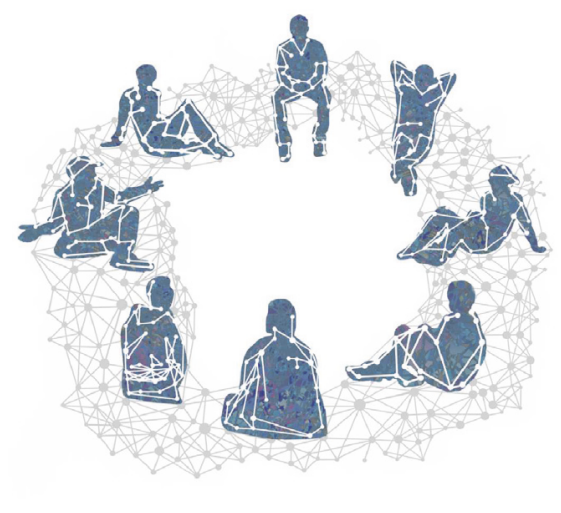

(b)

FIgure 1: Two of the works produced by J. K. Rofling and taken from [5] with permission of the artist. (a) The Trand. (b) The Guys.

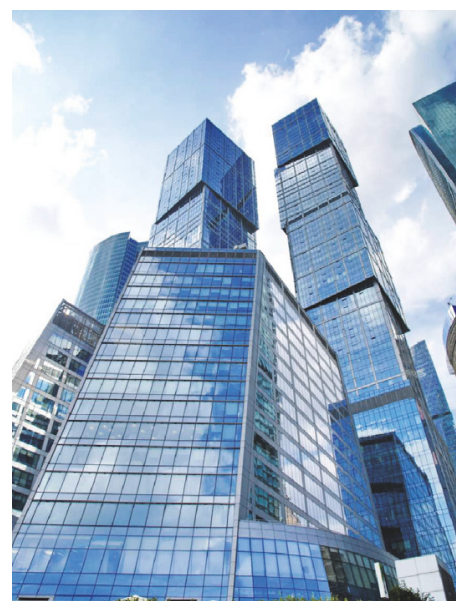

(a)
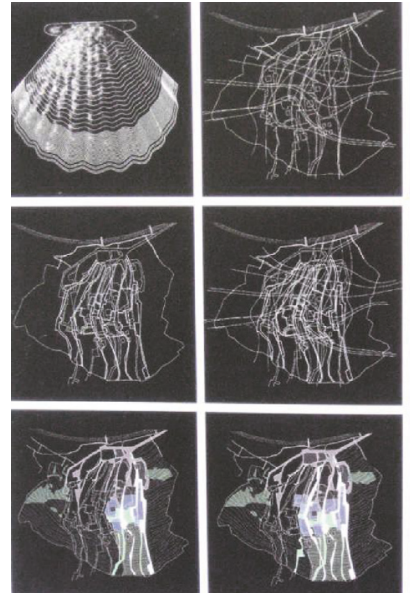

(b)

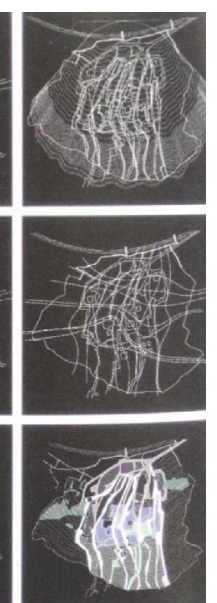

Figure 2: (a) City of Capitals in Moscow IBC, Russia. (b) Diagram of City of Culture of Galicia, Santiago de Compostela, Spain, by Peter Eisenman.

We do not pretend here to make a complete analysis of the deconstructivism in architecture but mainly of having a basic idea of its principles to be applied beyond its original frontiers. We then notice that the architectural deconstructivism looks initially as a fragmentation of the buildings which lack any visual logic. However, the deconstructing work accounts not only for this fragmentation but also for keeping a "memory" of the original composition in such a way that it "remembers" what it was in the beginning, that is, a building. In the City of Capitals in Moscow IBC, Russia, which is illustrated in Figure 2(a), the building is deconstructed into its unit block, that is, a cube, which is them "multiplied" to create again a tower with a different shape as the traditional ones. Another characteristic of deconstructivism is that the whole work must superimpose elements in such a way that "the design is produced, and the idea follows as its result." As described by Hoteit [7] one of these examples is the City of Culture of Galicia, Santiago de Compostela, Spain, designed by Eisenman. According to Hoteit [7] "Eisenman was mostly known for using the superimposition of layers." In his creation of the City of Culture of Galicia "Eisenman determined the following four local traces: The downtown's historical street grid; the typography of a hill; the abstract Cartesian grid; and the symbol of the city of Santiago, which is the scallop shell. Then, he superimposed these four abstracted traces to create an imaginary site condition, which became a real site for his project" (see also [8]). This idea is illustrated in Figure 2(b).

The connection with mathematical ideas here is evident. The superposition of layers can be imitated by the sum of parts and the compositional part can be obtained by multiplying the deconstructed parts such that we can recover certain "memory" from the original object. There are of course several ways of imitating these two characteristics of deconstructivism, but we have selected these two for the sake of mathematical convenience. This idea attempts to follow the existing line of connection between mathematical objects and 
visual arts. This includes among others knots $[9,10]$, mosaics and tiles [11, 12], Fourier series [13], topological tori [14], and fractal curves [15], all of which produce artistic patterns of undoubtful beauty by themselves.

It can also be argued that some works in the cubism movement show elements of deconstruction. Indeed, analytic cubism is seen as an influential stream for deconstructivism via the work of Frank Gehry. Analytical cubism includes important paints by Picasso, Braque, Metzinger, and others [16, 17]. Here, again, the principles of fragmenting, integrating, and superimposing are relevant in the analysis of these works [17]. Focusing only on these three principles to understand deconstruction is a clear oversimplification. However, we consider them here as the angular stone for what we will consider in the current work. Here, we are concerned with a formulation of deconstruction principles in mathematics.

\section{Deconstructing Integer-Based Functions}

Formulating deconstructivist principles for the whole of mathematics is a too ambitious project for a single paper. Instead, we focus here on integers and functions of integers. Then, the question is how to deconstruct an integer? The first idea should be to consider the individual digits of an integer as its building blocks. That is, for an integer $x$ written in a given base $b$, it is represented by

$$
x=a_{1} b^{n}+a_{2} b^{n-1}+a_{3} b^{n-2}+\cdots+a_{n-1} b+a_{n},
$$

where $a_{i} \in \mathbb{Z}$ are nonnegative integers, which can be considered as the building blocks of $x$. For instance, the building blocks of $x=2018$ are 2, 0,1 , and 8 . Here the "whole" is represented by the integer, which in architecture should be the tower. The blocks are the digits forming that whole, like the cubes in the tower.

Now, we should proceed to the "superposition of layers" part. Here, we simply consider the function that sums the digits of the integer $x$ in the base $b[18]$ :

$$
S_{b}(x)=\sum_{i=1}^{n} a_{i}=\sum_{k=0}^{\left\lfloor\log _{b} x\right\rfloor} \frac{1}{b^{k}}\left(x \bmod b^{k+1}-x \bmod b^{k}\right) .
$$

For instance, for $x=2018$, the integration will produce $S_{10}(x)=11$. These sequences for different bases $b$ are stored in the On-Line Encyclopaedia of Integer Sequences [19, 20]; for instance, A007953 is the sequence for $b=10$.

In order to complete the deconstruction of the integer we need the "recovery of the memory" of the original object. That is, we consider the product of the integer $x$ by $S_{10}(x)$ as the final deconstruction of the integer $x$ [21]:

$$
\widehat{x_{b}}=x \sum_{i=1}^{n} a_{i}
$$

In this way, we have that a given integer is first dismembered into its digits; then the digits are superimposed to each other as the different layers of the integer using the digit-sum function. Finally, we "recover" the memory of the original number by multiplying the integer by its digit-sum. Hereafter, we consider only the base $b=10$; thus $\widehat{x}=\widehat{x_{b}}$. Using this approach, the deconstructed integers "remember" something about their original numbers. For instance, $\widehat{19}=190, \widehat{28}=$ $280, \widehat{37}=370, \widehat{46}=460, \widehat{55}=550, \widehat{91}=910$, and $\widehat{82}=$ 820 (see sequence A117570 in [19]). However, it does not mean that $\widehat{x_{b}}$ is different for each integer. For instance, $\widehat{75}=\widehat{150}=$ 900 .

Let us now extend this approach to any function based on integers. Let $f(x)$ be a function of the number $x$, for example, $\sin (x)$. Then, the sum of digit-functions $\widehat{f(x)}: \mathbb{Z} \rightarrow \mathbb{R}$ as the function defined on the integers, such that

$$
\widehat{f(x)}=\left(f\left(a_{1}\right)+f\left(a_{2}\right)+\cdots+f\left(a_{n}\right)\right) f(x) .
$$

For negative integers $-x$, if the function $f(-x)$ exists, we define

$$
\widehat{f(-x)}=\left(f\left(a_{1}\right)+f\left(a_{2}\right)+\cdots+f\left(a_{n}\right)\right) f(-x) .
$$

We then consider the plot of pairs of functions $\widehat{f(t)}$ and $\widehat{g(t)}$ for the integers $t \leq n / 2$ such that

$$
\begin{aligned}
& x=\widehat{f(t)}, \\
& y=\widehat{g(t)} .
\end{aligned}
$$

If the functions $\widehat{f(t)}$ and $\widehat{g(t)}$ are also defined for negative arguments we obtain the corresponding transforms for $-n / 2 \leq t$. We are going to use these functions to build spatial networks as described in the next section.

\section{Building Spatial Networks}

In this section, we define our strategy for building spatial graphs based on the deconstruction of integer-functions. This strategy is based on the random geometric graphs (RGGs). Thus, we first explain the way in which RGGs are built. The RGG is defined by distributing uniformly and independently $n$ points in the unit $d$-dimensional cube $[0,1]^{d}[22]$. Hereafter we consider only the 2-dimensional case. Then, two points are connected by an edge if their Euclidean distance is at most $R$, which is a given fixed number known as the connection radius. That is, we create a disk of radius $R$ centered at each node, and every node inside that disk is connected to the central node as illustrated in Figure 3. A few important structural parameters of RGGs have been determined analytically in the literature (see, e.g., [22]).

Now, let us consider the process $T$ that generates $n$ points in the unit square according to the transforms of integerfunctions defined in the previous section. For instance, let us consider $-1000 \leq t \leq 1000$ and make the following transformation.

Transform $1\left(T_{1}\right)$.

$$
\begin{aligned}
& x=\widehat{t}, \\
& y= \begin{cases}\widehat{\sin t}, & t \leq 0 \\
-\widehat{\sin t}, & t>0 .\end{cases}
\end{aligned}
$$




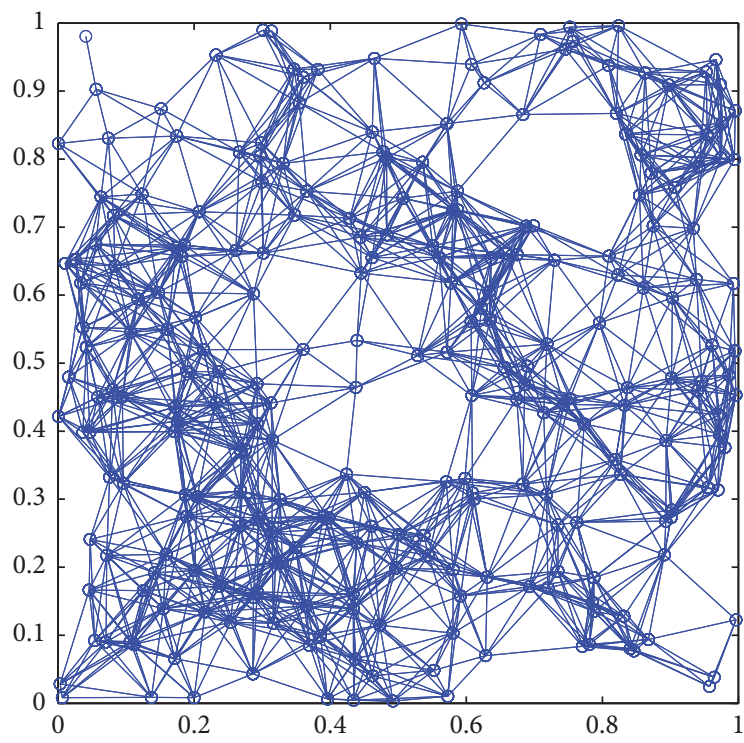

FIGURE 3: Illustration of a RGG created with 250 nodes embedded into a unit square where the nodes are connected if they are at a Euclidean distance smaller than or equal to $R=0.15$.

Notice that we consider the trigonometric functions of the numbers in degrees not in radians. For instance, $\sin t$ means "sine of $t$ degrees." Then, we plot every point on the unit square according to its coordinates $(x, y)$ defined before as illustrated in Figure 4(a). Using the approach to construct RGGs described before we construct the network for a given value of $R$. That is, after placing the points in the unit square we center a disk of radius $R$ on each point and connect to it every other point which is inside the corresponding disk. Here we will use radii which guarantee the connectivity of the graph-the study of the connectivity of these graphs is beyond the scope of the current work. For instance, in Figure 4(b) we illustrate the network created by using $R=$ 0.075 .

\section{Spatial "Artistic" Networks}

It is straightforward to realize that the previously obtained spatial graph (Figure 4(b)) displays a few artistic attributes: (i) biomorphic shape, that is, suggestive in shape of a living organism (a butterfly in this case); (ii) symmetry; and (iii) beauty, just to mention three. The appearance of a biomorphic shape here is just by chance and we have selected in this work only those transforms of integer-functions which produce artistically appealing shapes. However, it must be emphasized that both-beauty and interpretation of shapes-are on the eyes of the beholder, and different observers can see different things in these and other spatial networks created from integer-functions. Here we coin the name spatial "artistic" networks (SANs) for the spatial networks created using the previously described method.

Let us now consider other alternatives to the integerfunction deconstruction to see which artistic objects we can obtain. Artistic composition is the result of artist creativity and it includes a series of general rules that can be implemented computationally. Here, we mainly follow a handmade compositional creation in order to glue series of integer-function transforms into single art works. For instance, let us consider the following parametric equations.

Transform $2\left(T_{2}\right)$.

$$
\begin{aligned}
& x=\widehat{t}, \\
& y=\widehat{\cos t}+\widehat{t} \cdot \widehat{\sin t} .
\end{aligned}
$$

The resulting network for $R=0.085$ with $n=1000$ points is illustrated in Figure 5 where we have used $-500 \leq t \leq$ 500 and the nodes are colored according to their closeness centrality.

Transform $3\left(T_{3}\right)$. Another example is obtained by transforming the Astroid curve using the integer-digit transform. First, let us remind the reader that the Astroid is the curve: $x=$ $\cos ^{3}(t)$ and $y=\sin ^{3}(t)$. Then, we make the transformation of the coordinates as explained before, such that we have

$$
\begin{aligned}
& x=(\widehat{\cos t})^{3}, \\
& y=(\widehat{\sin t})^{3} .
\end{aligned}
$$

The corresponding SAN is illustrated in Figure 6, where we have used again $-1000 \leq t \leq 1000$ and the nodes are colored according to their closeness centrality.

Transform $4\left(T_{4}\right)$. The involute of the circle $-x=\cos t+$ $t \sin t ; y=\sin t-t \cos t$ - can also be transformed accordingly for $-1000 \leq t \leq 1000$ such that we obtain the following parametric equations:

$$
\begin{aligned}
& x=\widehat{\sin t}-\widehat{t} \widehat{\cos t}, \\
& y=-\widehat{\cos t}-\widehat{t} \widehat{\sin t},
\end{aligned}
$$

which produce the network illustrated in Figure 7.

Transform $5\left(T_{5}\right)$. Finally, we obtain the integer-function transformation of the cardioid curve, such that

$$
\begin{aligned}
& x=2 \widehat{\cos t}+\widehat{\cos (2 t)}, \\
& y=\frac{1}{2} \widehat{\cos t}-5 \widehat{\sin (2 t)},
\end{aligned}
$$

where

$$
\begin{aligned}
& \widehat{\cos (2 t)} \\
& \quad=\left(\cos \left(2 a_{1}\right)+\cos \left(2 a_{2}\right)+\cdots+\cos \left(2 a_{n}\right)\right) \cos (2 t) \\
& \widehat{\sin (2 t)} \\
& \quad=\left(\sin \left(2 a_{1}\right)+\sin \left(2 a_{2}\right)+\cdots+\sin \left(2 a_{n}\right)\right) \sin (2 t),
\end{aligned}
$$

with $t=a_{1} b^{n}+a_{2} b^{n-1}+a_{3} b^{n-2}+\cdots+a_{n-1} b+a_{n}$ represented in the decimal basis $b=10$. The spatial graph based on this transformation is illustrated in Figure 8 where we have used $-1000 \leq t \leq 1000$. 


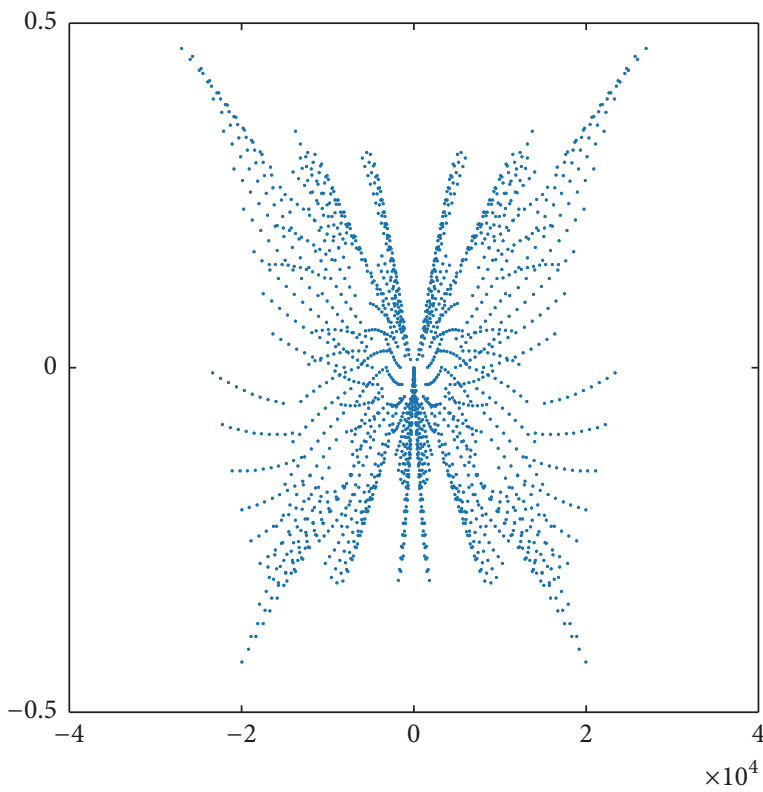

(a)

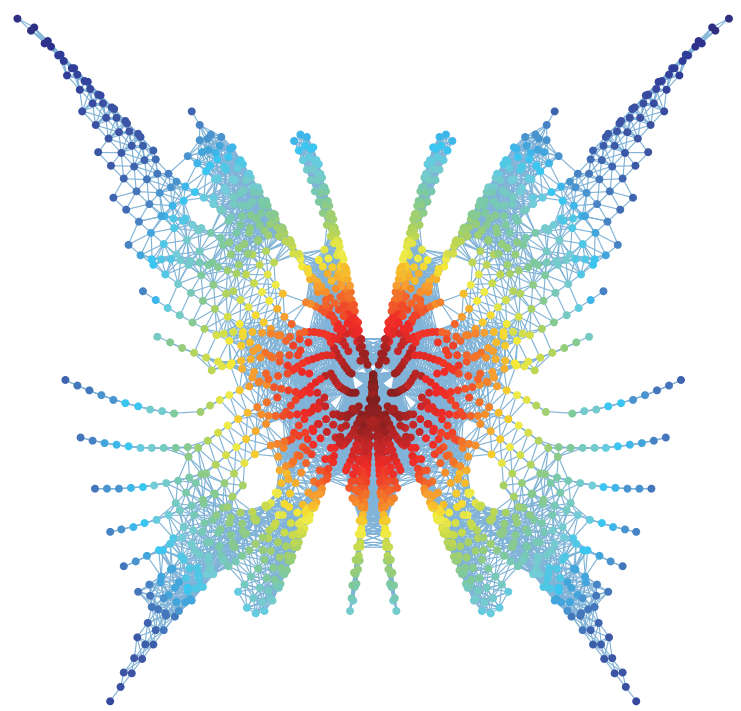

(b)

FIGURE 4: Illustration of the process to build a spatial network based on integer-function deconstruction. (a) Distribution of the points obtained from the transform $T_{1}$ on a square. (b) Construction of the spatial graph using a connection radius $R=0.075$ with $n=2,000$ points and coloring the nodes according to their closeness centrality.

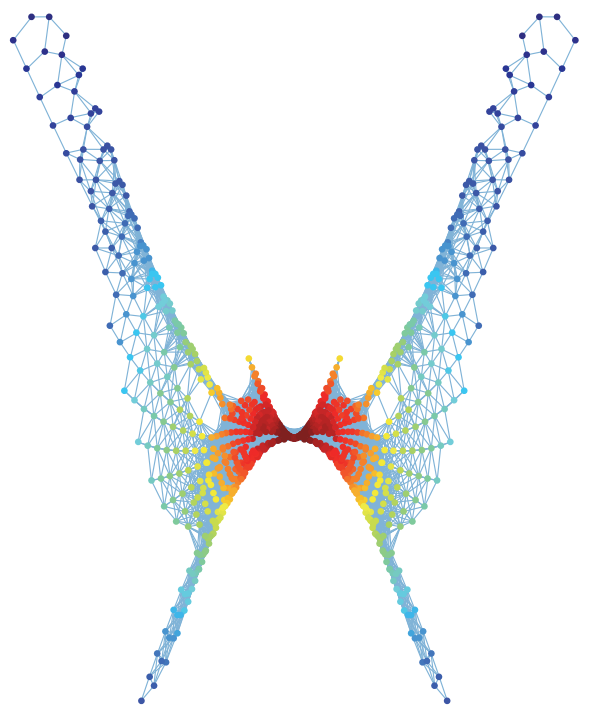

FIgURE 5: Spatial network constructed from the distribution of points in a unit square according to the transform $T_{2}$ using a connection radius $R=0.085$ with $n=1000$ points and coloring the nodes according to their closeness centrality.

\section{Network Invariants of SANs}

Here we consider a few invariants of the networks constructed by using the five transformations previously studied and compare them with the same invariants for the analogous RGG. That is, we construct RGGs with the same number of nodes and connection radius than the SANs created by the previously defined transforms. These invariants are as

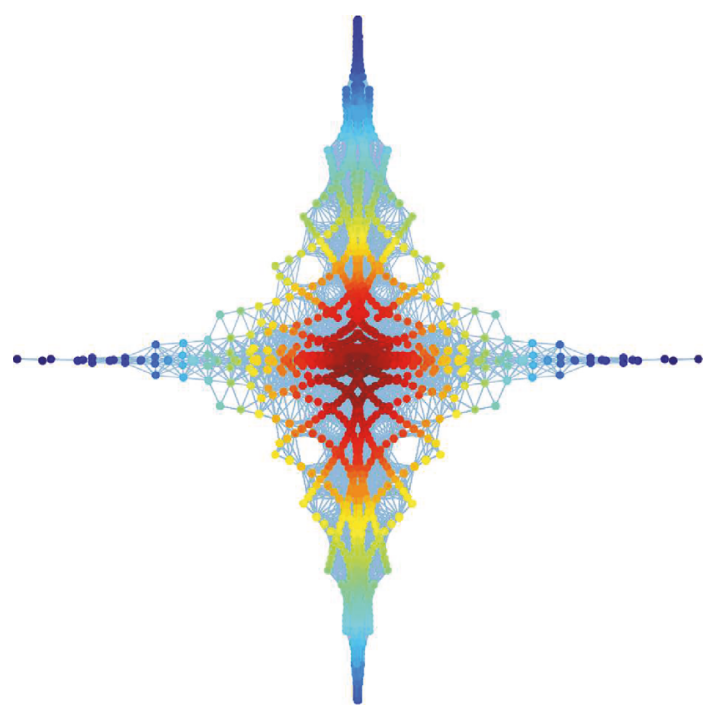

FIgURE 6: Spatial network constructed from the distribution of points in a unit square according to the transform $T_{3}$ using a connection radius $R=0.1$ with $n=2000$ points and coloring the nodes according to their closeness centrality.

follows: the number of nodes $n$, the number of edges $m$, the edge density $\delta$, the maximum degree $k_{\max }$, the average WattsStrogatz clustering coefficient $\bar{C}$, the global transitivity index $C$, average shortest path distance $\bar{d}$, network diameter $d_{\max }$, and the degree assortativity $r$ (for definitions and meaning see [23]). In Table 1, we give the values of these graph-theoretic invariants for the SANs and RGGs studied here. 
TABLE 1: Graph-theoretic invariants of the spatial "artistic" networks described in Section 4.

\begin{tabular}{|c|c|c|c|c|c|c|c|c|c|c|}
\hline & \multicolumn{2}{|c|}{$T_{1}$} & \multicolumn{2}{|c|}{$T_{2}$} & \multicolumn{2}{|c|}{$T_{3}$} & \multicolumn{2}{|c|}{$T_{4}$} & \multicolumn{2}{|c|}{$T_{5}$} \\
\hline & SAN & RGG & SAN & RGG & SAN & RGG & SAN & RGG & SAN & RGG \\
\hline$R$ & \multicolumn{2}{|c|}{0.075} & \multicolumn{2}{|c|}{0.085} & \multicolumn{2}{|c|}{0.1} & \multicolumn{2}{|c|}{0.085} & \multicolumn{2}{|c|}{0.1} \\
\hline$n$ & \multicolumn{2}{|c|}{2,000} & \multicolumn{2}{|c|}{1,000} & \multicolumn{2}{|c|}{2,000} & \multicolumn{2}{|c|}{2,000} & \multicolumn{2}{|c|}{2,000} \\
\hline$m$ & 30,158 & 33,128 & 32,286 & 10,440 & 129,656 & 57,287 & 198,951 & 42,113 & 61,217 & 57,287 \\
\hline$\delta$ & 0.015 & 0.017 & 0.077 & 0.021 & 0.065 & 0.029 & 0.099 & 0.021 & 0.031 & 0.029 \\
\hline$k_{\max }$ & 117 & 55 & 205 & 39 & 299 & 87 & 581 & 64 & 150 & 87 \\
\hline $\bar{C}$ & 0.618 & 0.613 & 0.665 & 0.608 & 0.727 & 0.622 & 0.642 & 0.614 & 0.695 & 0.622 \\
\hline$C$ & 0.217 & 0.200 & 0.249 & 0.198 & 0.247 & 0.202 & 0.264 & 0.202 & 0.225 & 0.202 \\
\hline $\bar{d}$ & 11.16 & 8.22 & 7.38 & 7.59 & 7.45 & 6.14 & 6.08 & 7.266 & 11.90 & 6.14 \\
\hline$d_{\max }$ & 46 & 21 & 39 & 19 & 23 & 15 & 34 & 18 & 33 & 15 \\
\hline$r$ & 0.65 & 0.60 & 0.75 & 0.59 & 0.74 & 0.61 & 0.79 & 0.60 & 0.68 & 0.61 \\
\hline
\end{tabular}

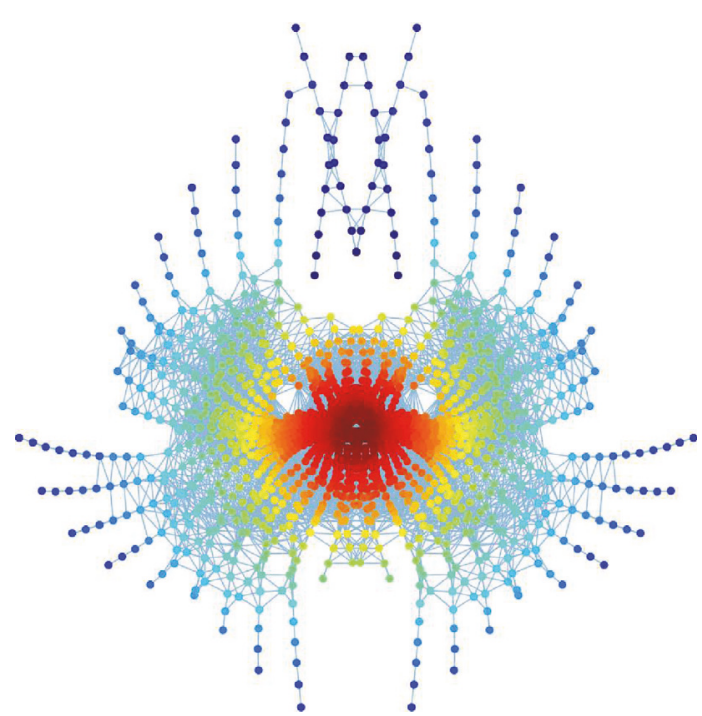

FIGURE 7: Spatial network constructed from the distribution of points in a unit square according to the transform $T_{4}$ using a connection radius $R=0.085$ with $n=2000$ points and coloring the nodes according to their closeness centrality.

In general, the graph-theoretic properties of SANs are relatively similar to those of the RGGs. However, there are some differences, particularly for the maximum degree and maximum distance. That is, the SANs always have significantly larger $k_{\max }$ and $d_{\max }$ than the corresponding RGGs. These two parameters are larger in the SANs as a consequence of the higher concentration of points in the center of the figure in relation to their peripheries. This situation is avoided in the RGG due to the random and homogeneous distributions of the points in the unit square. The similarities in terms of clustering coefficients and assortativity-notice that all networks are degree assortative-between SANs and RGGs are remarkable. We, however, are not claiming any application of these graphs for solving problems in the realworld, apart from being a source of artistic inspiration. Then, the analysis of these properties is mostly a curiosity-driven one and not the search for useful properties of these graphs. In the next section, we explore how these networks inspire some art.

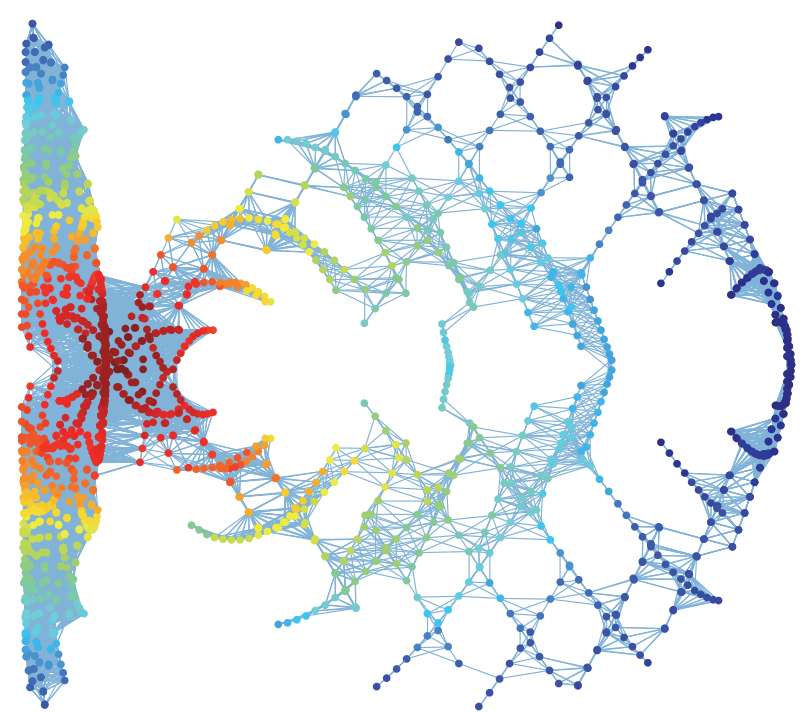

FIGURE 8: Spatial network constructed from the distribution of points in a unit square according to the transform $T_{5}$ using a connection radius $R=0.1$ with $n=2,000$ points and coloring the nodes according to their closeness centrality.

\section{Artistic Inspiration}

Science is sometimes seen as a dry and cold activity, such that it is not able to inspire those which are not involved in it. In earlier definitions of the humanities as "the branches of polite learning, especially the ancient classics and literature of aesthetics, as distinguishes from informational or utilitarian values" the sciences are marginalized as "informational but unaesthetic, that is, as useful but grubby" [24]. Many efforts are currently done for attracting the attention of the general public to the beauty of scientific discoveries. In mathematical sciences, for instance, there are initiatives, such as Bridges $[25,26]$, which bring together mathematicians and artists to produce artistic works from, or inspired by, mathematics. The Journal of Humanistic Mathematics [27] has also been launched to fill the gap between the humanities and mathematics. 


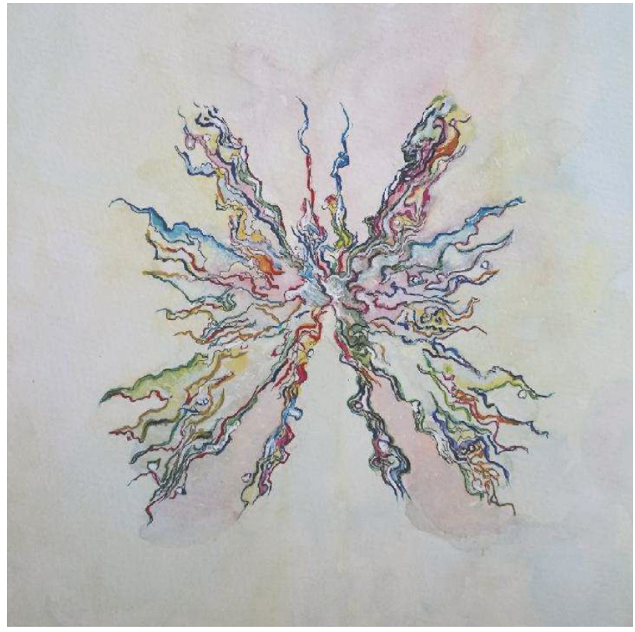

(a)

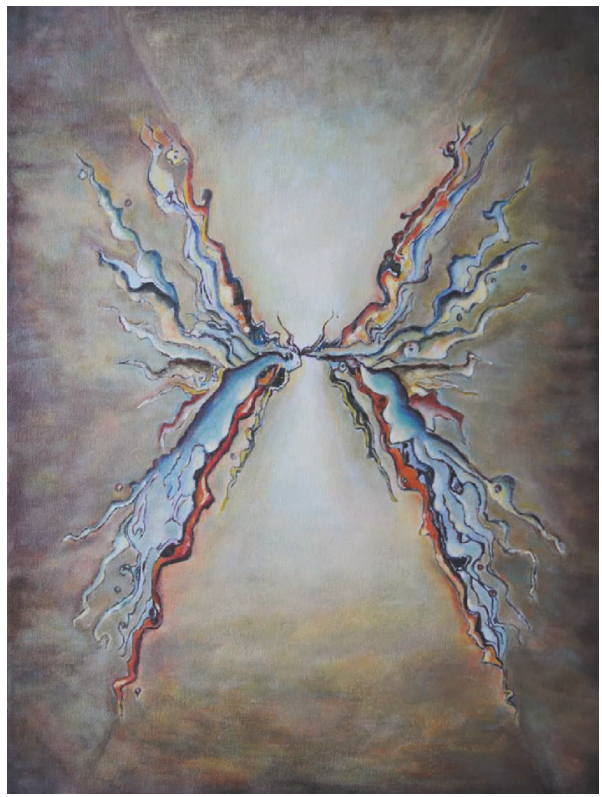

(c)

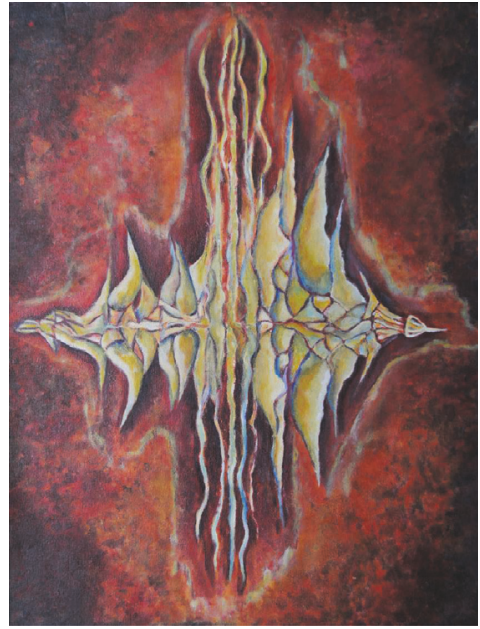

(b)

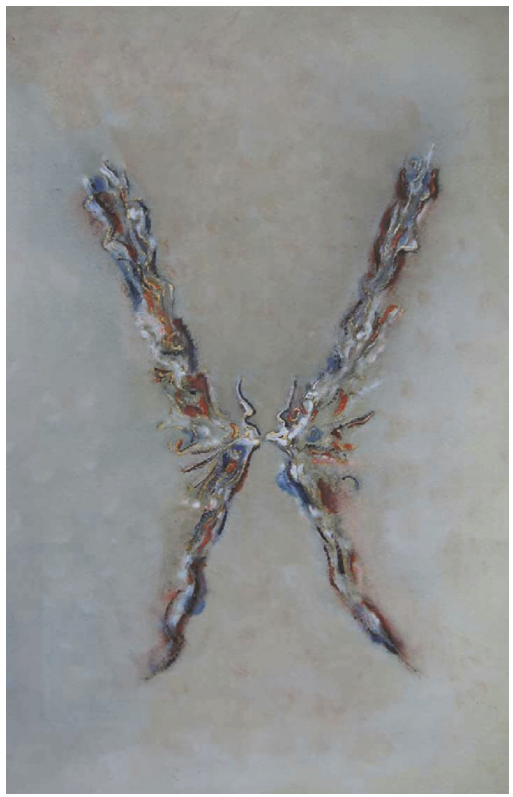

(d)

Figure 9: Photograph of four artistic works of artist Puri Pereira. (a) "Butterfly \# 1" painted in acrylic, ink, and watercolor on paper of dimensions $30 \times 40.5 \mathrm{~cm}$. (b) "Fire on Water" painted in acrylic on canvas of dimensions $45.7 \times 61 \mathrm{~cm}$. (c) "Butterfly \# 2" painted in acrylic on canvas of dimensions $45.7 \times 61 \mathrm{~cm}$. (d) "Birds" painted in ink, watercolor, and acrylic on paper of dimensions $30 \times 40.5 \mathrm{~cm}$.

In this part of our work, we present a few snapshots of what an artist can bring from the visual images produced by the SANs obtained from the integer-functions deconstruction presented here. That is, the SANs previously described have been the source of artistic inspiration for the production of purely aesthetic works outside the constraints of (network) sciences. The results are illustrated in Figure 9.

\section{On the Artistic Value of (Network) Sciences}

Network sciences have an important impact on our understanding of nature and modern society. Its practical importance has been documented in many papers in the last few years. But network science is also driven by aesthetic criteria.
Sometimes it is the mathematical beauty of the equations describing the structure of, or the dynamics on, the networks. Sometimes it is the beauty of the embedding of the network into certain space that produces outstanding visualizations. Other times it is the result of the application of network theory to a particular problem that produces an aesthetic feeling due to the beauty of the findings or what is unexpected of the connections found. Then, the importance of the applications of networks to solve practical problems should not hide its inherent beauty. The applicability of (network) sciences should not go in detriment of curiosity-driven, and aesthetic-driven, researches. We should find a compromise between application-driven and curiosity-driven researches. Abraham Flexner [28] - who was a founder of the Institute of 
Advanced Studies in Princeton and its Director from 1930 to 1939-stressed that "institutions of learning should be devoted to the cultivation of curiosity and the less they are deflected by considerations of immediacy of application, the more likely they are to contribute not only to human welfare but to the equally important satisfaction of intellectual interest which may indeed be said to have become the ruling passion of intellectual life in modern times." Obviously, there are many pressing problems in modern society that we are aimed to solve using network methods and approaches, and we should never forget our social responsibility. But our institutions should not forget either that as Flexner remarked "a poem, a symphony, a painting, a mathematical truth, a new scientific fact, all bear in themselves all the justification that universities, colleges, and institutes of research need or require" [28]. Thus, we should be reminded that (network) science has a humanistic side, which is as important as the many applications that it has found. Forgetting this side of it-its beauty and capacity of surprising -is similar to tear a wing to a bird. We all know that birds with only one wing cannot fly.

\section{Conclusions}

The spatial artistic networks (SANs) created here are the product of a curiosity-driven process more than of any practical necessity or real-world application. Thus, the value of these networks does not reside in their usefulness as a mathematical tool for modeling reality but as a source of inspiration of artistic work as well as attractive objects per se. We do not discard, however, that such networks can find some applications for modeling spatial processes in the real-world, due to their similarities with RGGs as well as by the fact that the points here are not randomly distributed in space but by using well-defined mathematical rules. The type of highdensity core and very sparse periphery reminds one with the situation frequently found in many spatial networks like cities.

Many chemistry students have been motivated to their subject by the beauty of the representations of the molecular structure. In physics, a similar situation exists when we consider the aesthetic of cosmic landscapes and the structure of the universe. Mathematicians always claim to be seduced by the beauty of mathematical equations. Can we attract students into network sciences by appealing to the aesthetic beauty of networks? The only way to know it is by trying. We hope that the current work contributes to this goal, either by attracting curious minds to the field or by inspiring other researchers in the field to explore the beauty of networks per se.

\section{Conflicts of Interest}

The authors declare that they have no conflicts of interest regarding the publication of this paper.

\section{References}

[1] H. C. Purchase, R. F. Cohen, and M. James, "Validating graph drawing aesthetics," in International Symposium on Graph Drawing, vol. 1027 of Lecture Notes in Computer Science, pp. 435-446, Springer, Berlin, Heidelberg, 1995.
[2] H. C. Purchase, "Metrics for graph drawing aesthetics," Journal of Visual Languages and Computing, vol. 13, no. 5, pp. 501-516, 2002.

[3] M. De Domenico, M. A. Porter, and A. Arenas, "MuxViz: A tool for multilayer analysis and visualization of networks," Journal of Complex Networks, vol. 3, no. 2, pp. 159-176, 2015.

[4] http://tomassaraceno.com/.

[5] https://www.jkrofling.com/.

[6] J. Derrida, "De la Grammatologie," de Minuit, 1967.

[7] A. Hoteit, "Deconstructivism: Translation From Philosophy to Architecture," Canadian Social Science, vol. 11, pp. 117-129, 2015.

[8] V. Belogolovsky, "One-on-one: Architecture that leads to a point: Interview with Daniel Libeskind," pp. 10-12, 2016, http://www.archnewsnow.com/features/Feature369.htm.

[9] A. Åström and C. Åström, "Circular knotworks consisting of pattern no. 295: a mathematical approach," Journal of Mathematics and the Arts, vol. 5, no. 4, pp. 185-197, 2011.

[10] R. Bosch, "Simple-closed-curve sculptures of knots and links," Journal of Mathematics and the Arts, vol. 4, no. 2, pp. 57-71, 2010.

[11] R. Bosch and U. Colley, "Figurative mosaics from flexible Truchet tiles," Journal of Mathematics and the Arts, vol. 7, no. 3-4, pp. 122-135, 2013.

[12] X. Zheng and N. S. Brown, "Symmetric designs on hexagonal tiles of a hexagonal lattice," Journal of Mathematics and the Arts, vol. 6, no. 1, pp. 19-28, 2012.

[13] F. A. Farris, "Symmetric yet organic: Fourier series as an artist's tool," Journal of Mathematics and the Arts, vol. 7, no. 2, pp. 6482, 2013.

[14] C. H. Séquin, "Topological tori as abstract art," Journal of Mathematics and the Arts, vol. 6, no. 4, pp. 191-209, 2012.

[15] J. Briggs, The patterns of chaos: A new aesthetic of art, science, and nature, A Touchstone Book, Simon \& Schuster, USA, 1992.

[16] R. L. Taylor, "Cubism - Abstract or Realist?," Philosophy and the Visual Arts," in Cubism- Abstract or Realist, pp. 77-95, Springer, Netherlands, Amsterdam, 1987.

[17] D. A. Gall, "Fragments of what? Postmodernism, Hybridity and Collage," Journal of Art for Life, vol. 5, p. 24, 2014.

[18] L. E. Bush, "An asymptotic formula for the average sum of the digits of integers," The American Mathematical Monthly, vol. 47, pp. 154-156, 1940.

[19] N. J. Sloane and S. Plouffe, The Encyclopedia of Integer Sequences, Academic Press, 1995, http://oeis.org.

[20] N. J. Sloane, "My favorite integer sequences," in Sequences and their applications, C. Ding, T. Helleseth, and H. Niederreiter, Eds., pp. 103-130, Springer, 1998.

[21] E. Estrada and L. A. Pogliani, "A new integer sequence based on the sum of digits of integers," Kragujevac Journal of Sciences, vol. 30, pp. 45-50, 2008.

[22] M. D. Penrose, Random Geometric Graphs, Oxford University Press, 2003.

[23] E. Estrada, The Structure of Complex Networks: Theory and applications, Oxford University Press, 2012.

[24] F. J. Rutherford, "A Humanistic Approach to Science Teaching," NASSP Bulletin, vol. 56, no. 361, pp. 53-62, 1972.

[25] K. Fenyvesi, "Bridges: A World Community for Mathematical Art," The Mathematical Intelligencer, vol. 38, no. 2, pp. 35-45, 2016.

[26] http://www.bridgesmathart.org/.

[27] http://scholarship.claremont.edu/jhm/.

[28] A. Flexner, The Usefulness of Useless Knowledge, Harper's Magazine, 1939. 


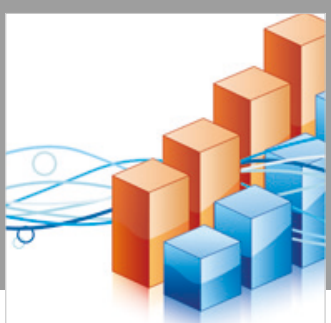

Advances in

Operations Research

\section{-n-m}
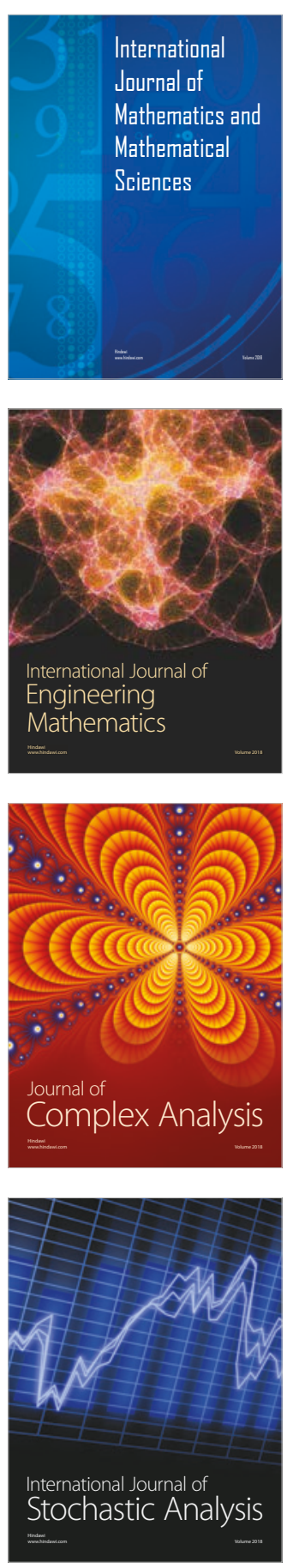
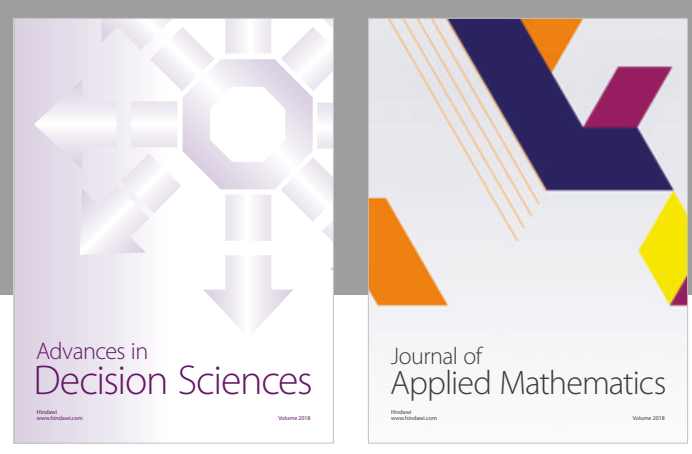

Journal of

Applied Mathematics
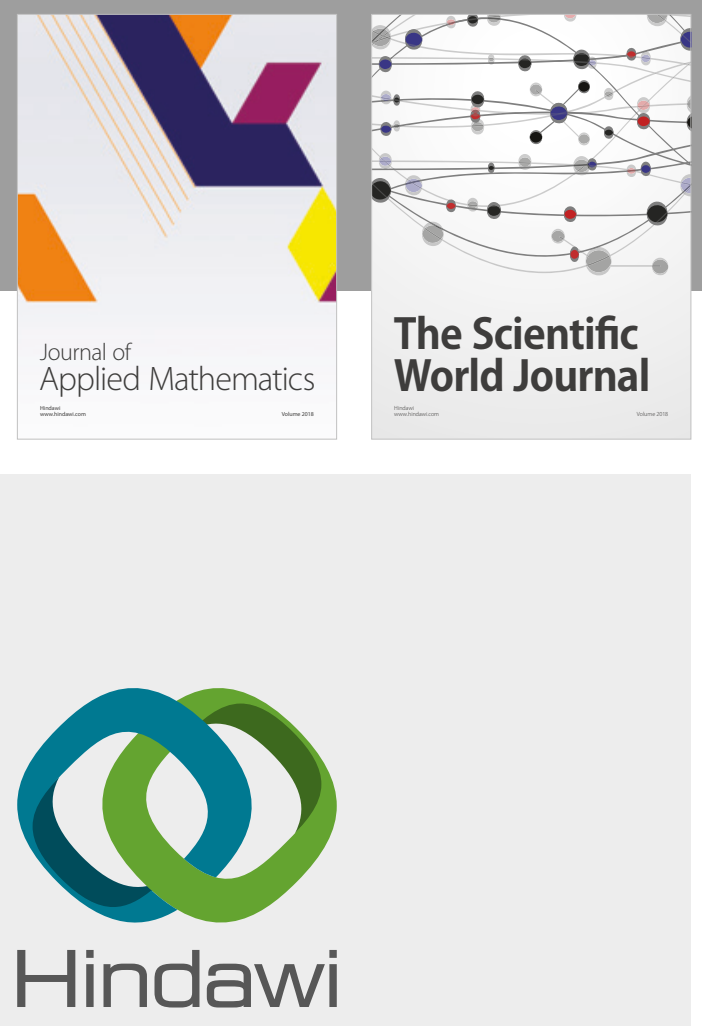

Submit your manuscripts at

www.hindawi.com

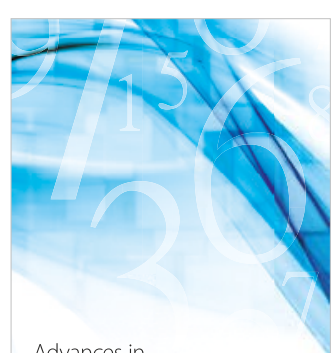

Advances in
Numerical Analysis
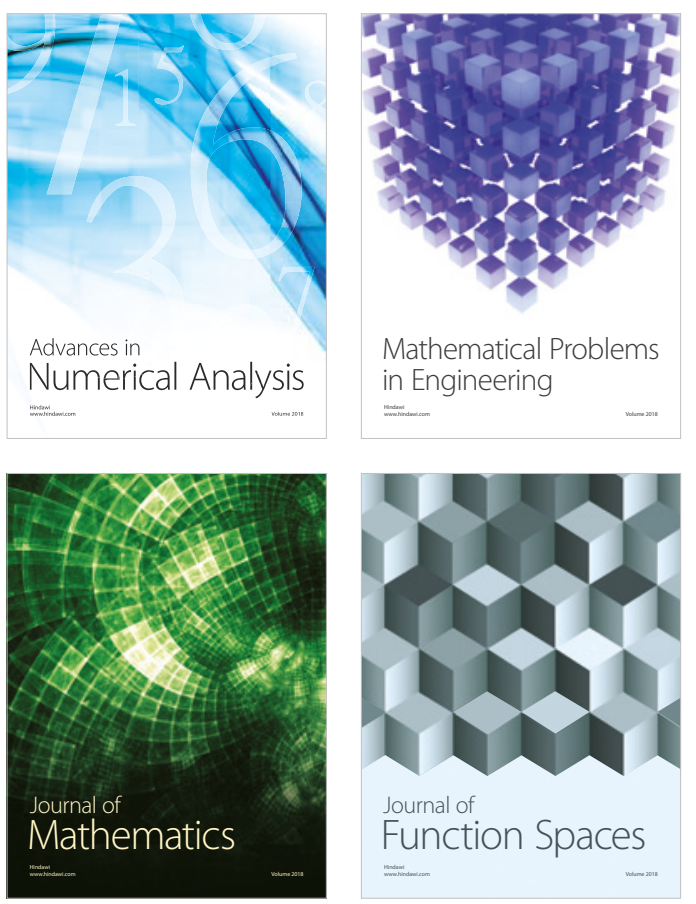

Mathematical Problems in Engineering

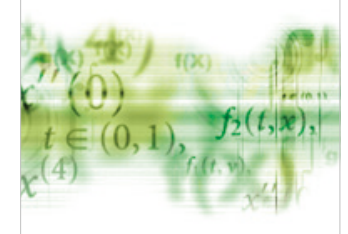

International Journal of

Differential Equations

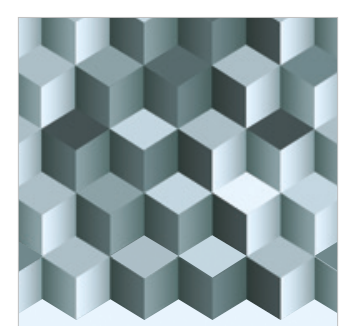

Journal of

Function Spaces
The Scientific

World Journal

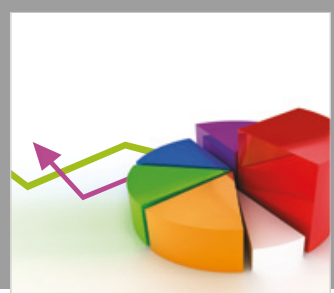

Journal of

Probability and Statistics
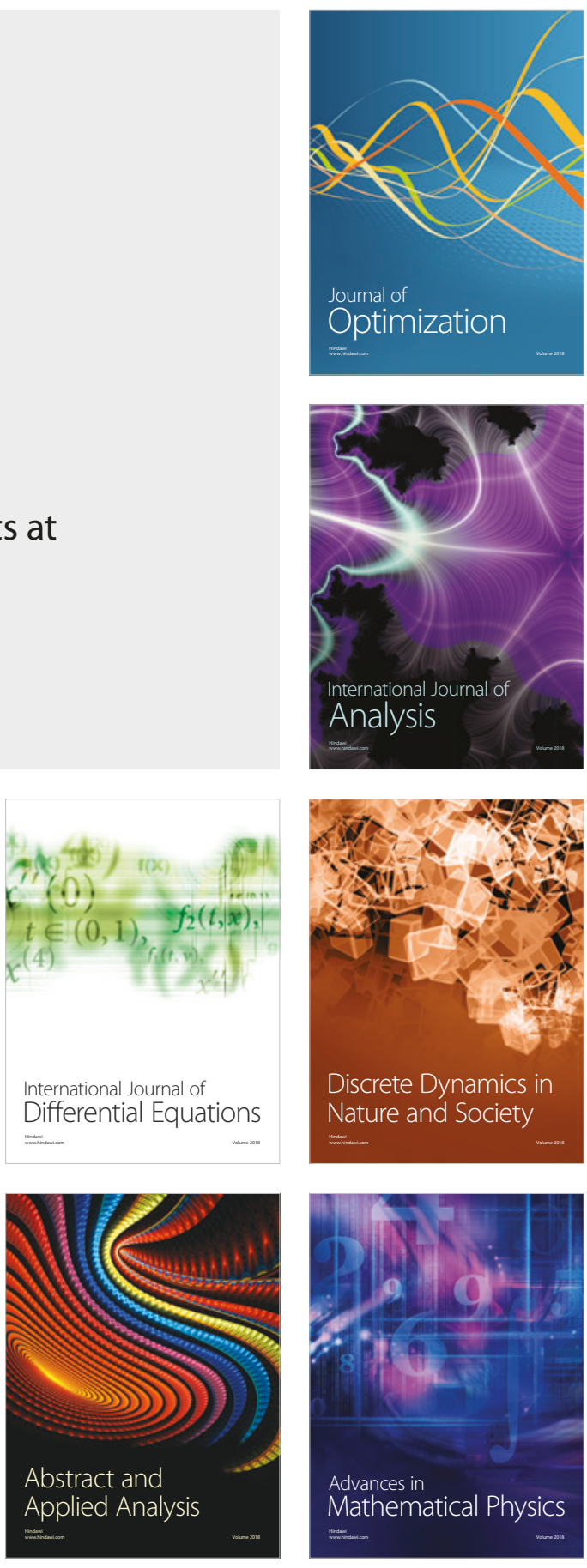